PROCEEDINGS OF THE

AMERICAN MATHEMATICAL SOCIETY

Volume 132, Number 9 , Pages 2549-2556

S 0002-9939(04)07390-3

Article electronically published on April 8, 2004

\title{
PURE PICARD-VESSIOT EXTENSIONS WITH GENERIC PROPERTIES
}

\author{
LOURDES JUAN
}

(Communicated by Lance W. Small)

\begin{abstract}
Given a connected linear algebraic group $G$ over an algebraically closed field $C$ of characteristic 0 , we construct a pure Picard-Vessiot extension for $G$, namely, a Picard-Vessiot extension $\mathcal{E} \supset \mathcal{F}$, with differential Galois group $G$, such that $\mathcal{E}$ and $\mathcal{F}$ are purely differentially transcendental over $C$. The differential field $\mathcal{E}$ is the quotient field of a $G$-stable proper differential subring $\mathcal{R}$ with the property that if $F$ is any differential field with field of constants $C$ and $E \supset F$ is a Picard-Vessiot extension with differential Galois group a connected subgroup $H$ of $G$, then there is a differential homomorphism $\phi: \mathcal{R} \rightarrow E$ such that $E$ is generated over $F$ as a differential field by $\phi(\mathcal{R})$.
\end{abstract}

\section{INTRODUCTION}

The notion of pure extensions has appeared in the study of whether a linear algebraic group over an algebraically closed field of characeristic 0 admits a generic linear differential equation. The subject was first studied by L. Goldman [3] who developed a theory of differential specialization and used it to define the concept of generic linear differential equation with group $G$ - the differential analogue of Noether's generic equation in the classical Galois theory of fields [7. Goldman explicitly constructed a generic equation with group $G$ for $G=\mathrm{GL}_{n}, \mathrm{SL}_{n}$, the reducible group consisting of all unimodular matrices $\left[a_{i j}\right]$ such that $a_{r+k, m}=0$, $k=1, \ldots, s, m=1, \ldots, r, r+s=n$, the orthogonal group, and the symplectic group. Later Bhandari and Sankaran [1] modified Goldman's conditions and produced a generic equation for the special orthogonal groups with weaker properties than Goldman's equation.

As was the case for Noether's theory, Goldman showed that if a linear algebraic group $G$ (assumed to be embedded in $\mathrm{GL}_{m}$ for some $m$ ) admits such a generic differential equation of order $m$, then the field of $G$-invariants of the differential field $C\left\langle\eta_{1}, \ldots, \eta_{m}\right\rangle$ of rational functions in the $m$ differential indeterminates $\eta_{i}$ over the field of constants $C$ is a purely differentially transcendental extension of $C$, generated as well by $m$ differential indeterminates $t_{1}, \ldots, t_{m}$. Moreover, it follows from Goldman's construction that $C\left\langle\eta_{1}, \ldots, \eta_{m}\right\rangle \supset C\left\langle t_{1}, \ldots, t_{m}\right\rangle$ is a Picard-Vessiot extension with differential Galois group $G$. We call such an extension where both

Received by the editors August 26, 2002 and, in revised form, June 2, 2003.

2000 Mathematics Subject Classification. Primary 12H05; Secondary 12F12, 20 G15.

The author was supported in part by NSA grant No. MDA904-02-1-0084. 
the base and the extension fields are purely differentially transcendental over the field of constants $C$, a pure Picard-Vessiot extension.

In this paper we show that pure Picard-Vessiot extensions with generic properties similar to those of Goldman's exist for connected linear algebraic groups. As in the case of Goldman and Bhandari-Sankaran, we will only work in characteristic 0 . We summarize our results as follows:

(1) Any connected linear algebraic group of dimension $n$ over the algebraically closed field of constants $C$ can be realized as a differential Galois group over $\mathcal{F}=C\left\langle Y_{1}, \ldots, Y_{n}\right\rangle$ where the $Y_{i}$ are differential indeterminates over $C$.

(2) The corresponding Picard-Vessiot extension $\mathcal{E} \supset \mathcal{F}$ has the form $\mathcal{E}=$ $\mathcal{F}\left(X_{1}, \ldots, X_{n}\right)$ where the $X_{i}$ are algebraically independent over $\mathcal{F}$ and the derivation on $\mathcal{E}=\mathcal{F}\left(X_{i}\right)$ is given by a "general element" of the extended Lie algebra $\mathcal{F} \otimes \mathfrak{g}$, where $\mathfrak{g}$ is the Lie algebra of $G$ (Section 2.1). Moreover, under this derivation, the $X_{i}$ are differentially independent over $C$ and $C\left\langle Y_{i}\right\rangle\left(X_{i}\right)$ equals $C\left\langle X_{i}\right\rangle$.

(3) The Picard-Vessiot extension $\mathcal{E} \supset \mathcal{F}$ is generic in the sense defined in Section 2.2

Since our results are true for all connected linear algebraic groups, they include and enlarge the class of groups for which it is known to have either a generic PicardVessiot extension or a generic equation with group $G$ [1, 3, 4, We also point out that for both Goldman and Bhandari-Sankaran, their constructions are based on the order $m$ of the equation and they are therefore attached to a representation of $G$ in $\mathrm{GL}_{m}$. In contrast, our construction is based on the dimension $n$ of the group and works for any faithful representation of $G$ in a $\mathrm{GL}_{m}$.

In [4] we proved that an extension with the properties above exists for the group $\mathrm{GL}_{n}$ as a consequence of a characterization of the principal differential ideals in the ring $F\left\{Y_{11}, \ldots, Y_{n n}\right\}\left[X_{11}, \ldots, X_{n n}\right]$, where the $Y_{i j}$ are differentially independent over $F$ and the $X_{i j}$ are algebraically independent over $F\left\langle Y_{11}, \ldots, Y_{n n}\right\rangle$, provided that $F$ is a differential field of characteristic 0 with algebraically closed field of constants $C$.

The approach followed in this paper is different and was inspired by a shorter proof of our construction for $\mathrm{GL}_{n}$ that Michael Singer pointed out to us (see Section 3). Here we further simplify Singer's proof and generalize it to all connected linear algebraic groups.

Our present method of producing a pure Picard-Vessiot extension is drastically simpler than the one used in 4. The drawback is that we do not get as much information since we do not know the differential semi-invariants (Darboux polynomials) in the ring $C\left\{Y_{i}\right\}\left[X_{i}\right]$ whose quotient ring is the generic extension.

The author wishes to thank Michael Singer for pointing out his proof and Andy Magid for his time and useful recommendations. The author is also grateful to the Kolchin Seminar in Differential Algebra and thanks Phyllis Cassidy, Jerry Kovacic, and William Sit for key observations that substantially improved the exposition of this manuscript.

\section{Definitions And BASIC RESUlts}

Note on the characteristic. All the fields considered in this paper are of characteristic 0 . 
2.1. G-equivariant derivations in $\mathcal{F}(\mathbf{G})$. We will discuss how, given a differential field $\mathcal{F}$ whose field of constants $C$ is algebraically closed and a connected linear algebraic group $G$ over $C$ of dimension $n$, one can express any $G$-equivariant derivation in the coordinate ring of the group $G_{\mathcal{F}}$, i.e., the group obtained from $G$ by extending scalars from $C$ to $\mathcal{F}$, in terms of the derivation on $\mathcal{F}$ and an element of the extended Lie algebra $\mathcal{F} \otimes_{C} \mathfrak{g}=\operatorname{Lie}\left(G_{\mathcal{F}}\right)$, where $\mathfrak{g}$ denotes the Lie algebra of $G$.

Write $\mathcal{R}$ for the coordinate ring $\mathcal{F}[G]=\mathcal{F} \otimes_{C} C[G]$ of $G_{\mathcal{F}}$. The quotient field $\mathcal{F}(G)$ of $\mathcal{R}$ is the function field of $G$. We observe that $\mathcal{R} \supset \mathcal{F}=\mathcal{F} \otimes 1$. Any $G$-equivariant derivation on $\mathcal{F}(G)$ over $\mathcal{F}$ is in the Lie algebra $\mathcal{F} \otimes \mathfrak{g}$ and hence preserves $\mathcal{R}$, making it a differential ring.

Now let $\mathcal{D}$ be any $G$-equivariant derivation on $\mathcal{R}$ with $\left.\mathcal{D}\right|_{\mathcal{F}}=\mathcal{D}_{\mathcal{F}} \otimes 1$, where $\mathcal{D}_{\mathcal{F}}$ is the derivation on $\mathcal{F}$. Define a derivation $\overline{\mathcal{D}}$ on $\mathcal{R}$ by $\overline{\mathcal{D}}=D_{\mathcal{F}} \otimes 1$. Then $\mathcal{D}-\overline{\mathcal{D}}$ is a $G$-equivariant derivation on $\mathcal{R}$ over $\mathcal{F} \otimes 1$ and therefore $\mathcal{D}-\overline{\mathcal{D}} \in \mathcal{F} \otimes \mathfrak{g}$; thus

$$
\mathcal{D}=\overline{\mathcal{D}}+\sum_{i=1}^{n} f_{i} \otimes \mathcal{D}_{i}
$$

where the $f_{i} \in \mathcal{F}$, the set $\left\{\mathcal{D}_{1}, \ldots, \mathcal{D}_{n}\right\}$ is a basis for $\mathfrak{g}$, and, consequently, $\{1 \otimes$ $\left.\mathcal{D}_{1}, \ldots, 1 \otimes \mathcal{D}_{n}\right\}$ is a basis for $\mathcal{F} \otimes \mathfrak{g}$. Now, if we pass to the quotient field $\mathcal{F}(G)$ of $\mathcal{R}=\mathcal{F} \otimes_{C} C[G]$, the restriction to $\mathcal{R}$ of any $G$-equivariant derivation which extends $\mathcal{D}_{\mathcal{F}} \otimes 1$, should be of the form of equation (11). Conversely, any $G$-equivariant derivation $\mathcal{D}$ on $\mathcal{F} \otimes_{C} C[G]$ such that $\left.\mathcal{D}\right|_{\mathcal{F}}=D_{\mathcal{F}} \otimes 1$, extends to its quotient field in a unique way by the quotient rule:

$$
\mathcal{D}\left(\frac{p}{q}\right)=\frac{\mathcal{D}(p) q-\mathcal{D}(q) p}{q^{2}} .
$$

We record the above discussion in the following

Proposition 2.1.1. Let $\mathcal{D}$ be a $G$-equivariant derivation in the quotient field $\mathcal{F}(G)$ of $\mathcal{F} \otimes_{C} C[G]$, with $\left.\mathcal{D}\right|_{\mathcal{F}}=D_{\mathcal{F}} \otimes 1$. Then for each basis $\mathcal{D}_{1}, \ldots, \mathcal{D}_{n}$ of $\mathfrak{g}$ there are $f_{1}, \ldots, f_{n} \in \mathcal{F}$ such that on $\mathcal{F} \otimes_{C} C[G]$,

$$
\mathcal{D}=\mathcal{D}_{\mathcal{F}} \otimes 1+\sum_{i=1}^{n} f_{i} \otimes \mathcal{D}_{i} .
$$

Now let $C$ be an algebraically closed field of constants, and let $Y_{1}, \ldots, Y_{n}$ be differentially independent over $C$. Put $\mathcal{F}=C\left\langle Y_{i}\right\rangle$. Since the $Y_{i}$ are differential indeterminates over $C$, the field of constants of $\mathcal{F}$ is $C$. Again, let $\mathcal{D}_{1}, \ldots, \mathcal{D}_{n}$ be a basis for $\mathfrak{g}$, the Lie algebra of the connected linear algebraic group $G$ over $C$. By the above discussion,

$$
\mathcal{D}=\mathcal{D}_{\mathcal{F}} \otimes 1+\sum_{\ell=1}^{n} Y_{\ell} \otimes \mathcal{D}_{\ell}
$$

is a $G$-equivariant derivation on $\mathcal{R}=\mathcal{F} \otimes_{C} C[G]$ that extends in a unique way to the function field $\mathcal{F}(G)$.

Suppose that $F$ is a differential field with field of constants $C$ and consider the coordinate ring $F[G]=F \otimes_{C} C[G]$. Proposition 2.1.1 implies that if $\widehat{\mathcal{D}}$ is any $G$-equivariant derivation on $F \otimes_{C} C[G]$, then there are $f_{i} \in F$ such that $\widehat{\mathcal{D}}=$ $\mathcal{D}_{F} \otimes 1+\sum_{\ell=1}^{n} f_{\ell} \otimes \mathcal{D}_{\ell}$. That is, $\widehat{\mathcal{D}}$ is the image of $\mathcal{D}$ in equation (2) via the differential homomorphism $\phi: C\left\{Y_{i}\right\} \otimes_{C} C[G] \rightarrow F \otimes_{C} C[G]$ given by $\phi\left(Y_{i} \otimes 1\right)=f_{i} \otimes 1$ and 
$\phi(1 \otimes \alpha)=1 \otimes \alpha$, for $\alpha \in C[G]$. Due to this property, we call $\mathcal{D}$ a general element of $\mathcal{F} \otimes \mathfrak{g}$.

Next we will see that if we choose a faithful representation of $G$ into $\mathrm{GL}_{m}$ for some $m$, the $\mathcal{D}_{i}$ can be identified with matrices in $\mathrm{M}_{m}(C)$. For that we invoke the following result $([9], 4.4 .7)$, which is true for linear algebraic groups in general (i.e., not necessarily connected). If $H$ is a closed subgroup of the linear algebraic group $\widetilde{H}$ over $C$, we denote by $J$ the ideal of $C[\widetilde{H}]$ consisting of functions that vanish on $H$, so that $C[H] \cong C[\widetilde{H}] / J$. Write $\mathcal{D}_{\widetilde{H}, H}$ for the set of $\widetilde{H}$-equivariant derivations $D$ of $C[\widetilde{H}]$ that satisfy $D(J) \subset J$. There is an obvious Lie algebra homomorphism

$$
\phi: \mathcal{D}_{\widetilde{H}, H} \rightarrow \mathcal{D}_{H},
$$

where $\mathcal{D}_{H}$ denotes the set of $H$-equivariant derivations on $C[H]$. We have:

Theorem 2.1.2. The homomorphism $\phi$ defines an isomorphism of $\mathcal{D}_{\widetilde{H}, H} \cap \widetilde{\mathfrak{h}}$ onto $\mathfrak{h}$, where $\widetilde{\mathfrak{h}}$ and $\mathfrak{h}$ are the Lie algebras of $\widetilde{H}$ and $H$, respectively.

If $G$ is a linear algebraic group, it is a closed subgroup of $\mathrm{GL}_{m}$ for some $m$. In this case, by Theorem 2.1.2 the Lie algebra $\mathfrak{g}$ of $G$ is isomorphic to a Lie subalgebra of the Lie algebra $\mathrm{M}_{m}$ of $\mathrm{GL}_{m}$ and the elements of $\mathfrak{g}$ can be identified with $m \times m$ matrices.

2.2. Generic Picard-Vessiot extension with group $G$. As before, $C$ will denote an algebraically closed field with trivial derivation and $\mathcal{F}$ a differential field with $C$ its field of contstants. Let $G$ be a linear algebraic group over $C$. We say that a differential field extension $\mathcal{E} \supset \mathcal{F}$ is a generic Picard-Vessiot extension for $G$ if

(i) $\mathcal{E} \supset \mathcal{F}$ is a Picard-Vessiot extension with differential Galois group $G$ and there are differential indeterminates $Y_{1}, \ldots, Y_{n}$ over $C$, such that for every faithful representation of $G$ in a $\mathrm{GL}_{m}(C)$, the matrix form of the differential equation for which $\mathcal{E} \supset \mathcal{F}$ is a Picard-Vessiot extension can be expressed in the form $X^{\prime}=A\left(Y_{i}\right) X$, where $A\left(Y_{i}\right) \in M_{m}\left(C\left\{Y_{i}\right\}\right)$.

(ii) Let $F$ be a differential field with field of constants $C$. Given a faithful representation of $G$ in a $\mathrm{GL}_{m}(C)$, a matrix $A \in \mathrm{M}_{m}(F)$, and a Picard-Vessiot extension $E \supset F$ for the equation $X^{\prime}=A X$ with differential Galois group $H \leq G$, where $H$ is connected, there are $f_{1}, \ldots, f_{n} \in F$ such that the matrix $A\left(f_{i}\right)$ obtained from $A\left(Y_{i}\right)$ in part (i) via the differential specialization $Y_{i} \mapsto f_{i}$ is gauge equivalent to $A$.

(iii) For any such well-defined specialization $Y_{i} \mapsto f_{i} \in F$ and a faithful representation of $G$ in a $\mathrm{GL}_{m}(C)$, the corresponding matrix $A\left(f_{i}\right) \in M_{m}(F)$ is the matrix for a linear differential equation $X^{\prime}=A\left(f_{i}\right) X$ giving rise to a Picard-Vessiot extension $E \supset F$ with differential Galois group $H \leq G$.

\section{THE GENERAL LiNEAR GROUP CASE}

We begin by presenting Michael Singer's shorter proof of the following result, which inspired the proof of our generalization to connected linear algebraic groups:

Theorem 3.0.1 (Theorem 3.3.1, 4]). Let $Y_{i j}, i, j=1, \ldots, n$, be differential indeterminates over the differential field $F$ with algebraically closed field of constants $C$ and let $X_{i j}, i, j=1, \ldots, n$, be algebraically independent over $F\left\langle Y_{i j}\right\rangle$. Write 
$X$ and $Y$ respectively for the matrices $\left[X_{i j}\right]$ and $\left[Y_{i j}\right]$, and extend the derivation on $F\left\langle Y_{i j}\right\rangle$ to $F\left\langle Y_{i j}\right\rangle\left(X_{i j}\right)$ by letting $\mathcal{D}(X)=Y X$, where $\mathcal{D}(X)=\left[\mathcal{D}\left(X_{i j}\right)\right]$. Then $F\left\langle Y_{i j}\right\rangle\left(X_{i j}\right) \supset F\left\langle Y_{i j}\right\rangle$ is a Picard-Vessiot extension with differential Galois group $\mathrm{GL}_{n}(C)$. This extension is generic in the following sense: If $E$ is a Picard-Vessiot extension of $F$ with group $\mathrm{GL}_{n}(C)$, it is known that $E \cong F\left(X_{i j}\right)$ and the derivation $\hat{\mathcal{D}}$ on $E$ extends the derivation on $F$ in such a way that $\hat{\mathcal{D}}(X)=A X$ where $A \in \mathrm{M}_{n}(F)$; then $E$ can be obtained as the quotient field of the image of a differential homomorphism $\phi: F\left\{Y_{i j}\right\}\left[X_{i j}\right] \rightarrow E$ with $\phi\left(Y_{i j}\right)=f_{i j}$ and $\phi\left(X_{i j}\right)=X_{i j}$.

Proof. Consider the field $K=F\left\langle X_{i j}\right\rangle$, where the $X_{i j}$ are differential indeterminates. Denote the derivation on $K$ by $D$ (it will sometimes also be denoted by '). Write $Y_{i j} \in K$ for the $(i, j)$-entry of the matrix $X^{\prime} X^{-1}$. We then have the matrix equation:

$$
X^{\prime}=Y X
$$

In particular, we have $D\left(X_{i j}\right)=\sum_{\ell=1}^{n} Y_{i \ell} X_{\ell j}$. Now one verifies the following:

(a) The constants of $K$ are precisely $C$, the constants of $F$. This follows from Proposition 2.1.2 in 4 .

(b) The elements $\left\{Y_{i j}\right\}$ are differentially independent; i.e., they are essentially differential indeterminates. If not, the differential transcendence degree (see [5], Definition 3.2.33 and Theorem 5.4.12) of $F\left\langle Y_{i j}\right\rangle$ over $F$ is less than $n^{2}$. Equation (3) implies that the (usual) transcendence degree of $K$ over $F\left\langle Y_{i j}\right\rangle$ is at most $n^{2}$, since $F\left\langle X_{i j}\right\rangle=F\left\langle Y_{i j}\right\rangle\left(X_{i j}\right)$. Thus we have that for each $(i, j)$, the elements $D^{n^{2}}\left(X_{i j}\right), \ldots, D\left(X_{i j}\right), X_{i j}$ must be algebraically dependent over $F\left\langle Y_{i j}\right\rangle$; i.e., each $X_{i j}$ is differentially algebraic over $F\left\langle Y_{i j}\right\rangle$. Therefore, the differential transcendence degree of $K$ over $F\left\langle Y_{i j}\right\rangle$ is 0 . This implies, under our assumption, by Proposition 5.3.13 and Theorem 5.4.12 in [5], that the differential transcendence degree of $K$ over $F$ would be less than $n^{2}$, a contradiction.

(c) $K$ is a $P V$ extension of $F\left\langle Y_{i j}\right\rangle$. This follows from equation (3) and the fact that there are no new constants.

(d) The Galois group of $K$ over $F\left\langle Y_{i j}\right\rangle$ is $\mathrm{GL}_{n}(C)$. Clearly the action of $\mathrm{GL}_{n}(C)$ on $K$ given by $\left[X_{i j}\right] \mapsto\left[X_{i j}\right] \sigma$ for $\sigma \in \mathrm{GL}_{n}(C)$ defines a differential automorphism of $K$ over $F\left\langle Y_{i j}\right\rangle$. So $\mathrm{GL}_{n}(C)$ is a subgroup of the Galois group. On the other hand, the Galois group is a subgroup of $\mathrm{GL}_{n}(C)$.

(e) The $\left\{X_{i j}\right\}$ are algebraically independent over $F\left\langle Y_{i j}\right\rangle$. This is because $K=$ $F\left\langle Y_{i j}\right\rangle\left(X_{i j}\right)$ and the transcendence degree of $K$ over $F\left\langle Y_{i j}\right\rangle$ is equal to the dimension of the Galois group.

(f) The field $K=F\left\langle Y_{i j}\right\rangle\left(X_{i j}\right)$ constructed above is the same as (i.e., isomorphic to) the field in Theorem 3.0.1 and it is a generic Picard-Vessiot extension for $\mathrm{GL}_{n}(C)$. The first statement follows from (b) and (e) above. The second statement is proved as in Theorem 3.3.1 in [4].

\section{Generic Picard-Vessiot extensions FOR CONNECTED LINEAR ALGEBRAIC GROUPS}

Throughout this section $C$ will denote an algebraically closed field with trivial derivation and $G$ a connected linear algebraic group over $C$ of dimension $n$. 


\subsection{Pure Picard-Vessiot extensions with group G.}

Definition 4.1.1. A Picard-Vessiot extension $\mathcal{E} \supset \mathcal{F}$ is said to be pure if both $\mathcal{E}$ and $\mathcal{F}$ are purely differentially transcendental over their common field of constants C.

We proceed to construct a pure Picard-Vessiot extension with differential Galois group $G$.

Let $Y_{1}, \ldots, Y_{n}$ be differential indeterminates over $C$ and put $\mathcal{F}=C\left\langle Y_{i}\right\rangle$. Extend scalars for $G$ from $C$ to $\mathcal{F}$ and consider the function field of the extended group $G_{\mathcal{F}}$. This extended function field has the form $\mathcal{F}(G)=\mathcal{F}\left(X_{i}\right)$ where the $X_{i}, i=1, \ldots, n$, are rational functions on $G$ that are algebraically independent over $\mathcal{F}$. The latter is the statement that connected linear algebraic groups are rational varieties, which follows from the structure of the large cell in the Bruhat decomposition (see, for example, [2]).

From Section 2, we know that $\mathcal{F}\left(X_{i}\right)$ can be made into a differential field by endowing it with the general derivation $\mathcal{D}=\mathcal{D}_{\mathcal{F}} \otimes 1+\sum_{i=1}^{n} Y_{i} \otimes \mathcal{D}_{i}$, where $\mathcal{D}_{1}, \ldots, \mathcal{D}_{n}$ are a basis of the Lie algebra $\mathfrak{g}$ of $G$. Moreover, if a faithful representation of $G$ into $\mathrm{GL}_{m}(C)$ is chosen for some $m$, then Theorem 2.1.2 gives an isomorphism $\phi$ of a Lie subalgebra of $\mathcal{F} \otimes \mathrm{M}_{m}(C) \cong \mathrm{M}_{m}(\mathcal{F})$ onto $\mathcal{F} \otimes \mathfrak{g}$. Under this isomorphism, each $1 \otimes \mathcal{D}_{i}$ can be identified with a matrix $A_{i} \in \mathrm{M}_{m}(C)$.

The advantage of considering such a representation of $G$ into $\mathrm{GL}_{m}(C)$ is that one can give a formula for the action of the derivation on the generators of $\mathcal{F}(G)$ over $\mathcal{F}$. Indeed, assuming a faithful representation of $G$ in $\mathrm{GL}_{m}(C)$, we have that $\mathcal{F}(G)=\mathcal{F}\left(X_{i j}\right)$ where the $m \times m$ matrix $\left[X_{i j}\right]$ is a generic point of $G_{\mathcal{F}}$. In this case, if we write $Y=\sum_{i=1}^{n} Y_{i} A_{i}$ and $X=\left[X_{i j}\right]$, we get the matrix equation

$$
\mathcal{D}(X)=X^{\prime}=Y X \text {. }
$$

Since, in turn, $\mathcal{F}\left(X_{i}\right)=\mathcal{F}\left(X_{i j}\right)$, the $X_{i}$ are rational functions of the $X_{i j}$ and the derivatives of the $X_{i}$ are obtained from those of the $X_{i j}$ by applying quotient and product rules as necessary. However, we will only fix such a representation of $G$ into $\mathrm{GL}_{m}(C)$ when a formula like equation (4) is needed for certain computations. We have:

Theorem 4.1.2. The differential field $C\left\langle Y_{i}\right\rangle\left(X_{i}\right)$ defined above is equal to $C\left\langle X_{i}\right\rangle$ and the $X_{i}$ are differentially independent over $C$. Therefore, the field of constants of $C\left\langle Y_{i}\right\rangle\left(X_{i}\right)$ is equal to $C$.

Proof. Choose a faithful representation of $G$ in $\mathrm{GL}_{m}(C)$ for some $m$, so that an equation such as (4) holds. From this equation it follows that

$$
Y=\sum_{i=1}^{n} Y_{i} A_{i}=X^{\prime} X^{-1},
$$

where $X=\left[X_{i j}\right]$ and the set $\left\{A_{1}, \ldots, A_{n} \mid A_{i} \in \mathrm{M}_{m}(C)\right\}$ is a basis for $\phi^{-1}(\mathfrak{g}) \subset$ $\mathrm{M}_{m}(C)$ where $\phi$ denotes the isomorphism of Theorem 2.1 .2

We claim that $Y_{i} \in C\left\langle X_{i j}\right\rangle$. Indeed, since the $A_{i} \in \mathrm{M}_{m}(C)$ are linearly independent over $C$, they can be extended to a basis $\mathcal{A}$ of $\mathrm{M}_{m}(C)$. Let $\mathcal{B}$ be a dual basis to $\mathcal{A}$ for the trace form, and write $B_{i}$ for the element of $\mathcal{B}$ corresponding to $A_{i}$. Then one has $\operatorname{tr}\left(A_{i} B_{j}\right)=\delta_{i j}$. Now, multilplying (5) through by $B_{k}$, for $k=1, \ldots, n$, and taking the trace of the resulting expression, one gets

$$
Y_{k}=\operatorname{tr}\left(X^{\prime} X^{-1} B_{k}\right) \in C\left\langle X_{i j}\right\rangle .
$$


By an observation preceeding Theorem 4.1.2 we have that $C\left\langle X_{i j}\right\rangle=C\left\langle X_{i}\right\rangle$. Equation (6) then implies that $C\left\langle Y_{i}\right\rangle\left(X_{i}\right)=C\left\langle X_{i}\right\rangle$.

We now show that the $X_{i}$ are differentially independent over $C$. If not, the differential transcendence degree of $C\left\langle X_{i}\right\rangle=C\left\langle Y_{i}\right\rangle\left(X_{i}\right)$ over $C$ is less than $n$. By construction the $Y_{i}$ are differentially independent over $C$, thus the differential transcendence degree of $C\left\langle Y_{i}\right\rangle$ over $C$ is $n$. The usual transcendence degree of $C\left\langle Y_{i}\right\rangle\left(X_{i j}\right)$ over $C\left\langle Y_{i}\right\rangle$ is at most $m^{2}$. This implies that for each $(i, j)$, the elements $D^{m^{2}}\left(X_{i j}\right), \ldots, D\left(X_{i j}\right), X_{i j}$ must be algebraically dependent over $C\left\langle Y_{i}\right\rangle$; thus each $X_{i j}$ is differentially algebraic over $C\left\langle Y_{i}\right\rangle$. Therefore, the differential transcendence degree of $C\left\langle Y_{i}\right\rangle\left(X_{i j}\right)$ over $C\left\langle Y_{i}\right\rangle$ is 0 . Since $C\left\langle Y_{i}\right\rangle\left(X_{i j}\right) \supset C\left\langle Y_{i}\right\rangle \supset C$, the latter implies that the differential transcendence degree of $C\left\langle Y_{i}\right\rangle\left(X_{i j}\right)=C\left\langle Y_{i}\right\rangle\left(X_{i}\right)=$ $C\left\langle X_{i}\right\rangle$ over $C$ is $n$ as well, contrary to our assumption.

Corollary 4.1.3. $\mathcal{E} \supset \mathcal{F}$ is a pure Picard-Vessiot extension with differential Galois group $G$.

Proof. Again, consider a faithful representation of $G$ into $\mathrm{GL}_{m}$ for some $m$. Then we have $\mathcal{E}=\mathcal{F}\left(X_{i j}\right)$, where the $m \times m$ matrix $\left[X_{i j}\right]$ is a generic point of $G_{\mathcal{F}}$, and $X^{\prime}=Y X$, as above. If we let $V$ denote the finite-dimensional vector space over $C$ spanned by the $X_{i j}$, clearly $G(V) \subseteq V$ and $\mathcal{E}=\mathcal{F}\langle V\rangle$. Since $\mathcal{E}=\mathcal{F}\left(X_{i j}\right)$ is the function field of the extended group $G_{\mathcal{F}}$, one also has that the elements of $G$ act on $\mathcal{E}$ as differential automorphisms and $\mathcal{E}^{G}=\mathcal{F}$. From Theorem 4.1.2 we know that the extension has no new constants and therefore it is a Picard-Vessiot extension. Theorem 4.1.2 implies as well that $\mathcal{E} \supset \mathcal{F}$ is a pure Picard-Vessiot extension.

4.2. Generic extensions for connected linear algebraic groups. We will now show that the above pure Picard-Vessiot extension $\mathcal{E} \supset \mathcal{F}$ satisfies the definition of generic extension given in Section 2.

Theorem 4.2.1. $\mathcal{E} \supset \mathcal{F}$ is a generic Picard-Vessiot extension with differential Galois group $G$.

Proof. Part (i) is obvious, for if we choose a faithful representation of $G$ in $\mathrm{GL}_{m}(C)$ and let $A\left(Y_{i}\right)=Y$, then equation (4) above becomes a matrix equation for which $\mathcal{E} \supset \mathcal{F}$ is a Picard-Vessiot extension with differential Galois group $G$. For Part (ii), let $F$ be a differential field with field of constants $C$ and assume that there is a faithful representation of $G$ in a $\mathrm{GL}_{m}(C)$. Let $E \supset F$ be a Picard-Vessiot extension with differential Galois group $H \leq G$, where $H$ is connected, for the matrix equation $X^{\prime}=A X$, with $A \in \mathrm{M}_{m}(F)$. Since $H$ is connected, Kolchin's Main Structure Theorem of Picard-Vessiot Extensions (see [6]) implies that there is an isomorphism between $E$ and the function field $F(H)$ of $H_{F}$, as $D$-modules and as $H$-modules. If we identify $E$ with $F(H)$ under this isomorphism, then $E$ is the quotient field of the coordinate $\operatorname{ring} F \otimes_{C} C[H]$.

By Proposition 2.1.1 and Theorem 2.1.2 one has that any $H$-equivariant derivation on $F \otimes_{C} C[H]$ has the form

$$
\mathcal{D}=\mathcal{D}_{F} \otimes 1+\sum_{i=1}^{n} f_{i} \otimes \mathcal{D}_{i}
$$

where the $f_{i} \in F$ and $\mathcal{D}_{1}, \ldots, \mathcal{D}_{n}$ forms a basis of $\mathfrak{g}$. Moreover, one can choose $\mathcal{D}_{1}, \ldots, \mathcal{D}_{n}$ so that $\mathcal{D}_{1}, \ldots, \mathcal{D}_{n}$ corresponds to the matrix basis $A_{1}, \ldots, A_{n}$ of $\phi^{-1}(\mathfrak{g})$ $\subset \mathrm{M}_{m}(C)$ found in the proof of Theorem 4.1.2 Then, $Y=\sum_{i=1}^{n} Y_{i} A_{i}$. 
Let $\eta$ be a fundamental matrix for the equation $X^{\prime}=A X$ so that the PicardVessiot extension $E \supset F$ is generated over $F$ by the entries of $\eta$ and its derivatives. We have $\eta^{\prime}=A \eta$. Since the derivation on $E$ extends the derivation on $F$ and is determined by $D(\eta)=A \eta$, it follows that $A$ is gauge equivalent to a matrix $\tilde{A}$ in the Lie algebra $\phi^{-1}(\mathfrak{h}) \subset \phi^{-1}(\mathfrak{g})$. For this $\tilde{A}$ there are $f_{1}, \ldots, f_{n} \in F$ such that $\tilde{A}=\sum_{i=1}^{n} f_{i} A_{i}=A\left(f_{i}\right)$. The matrix $A\left(f_{i}\right)$ is obtained from $A\left(Y_{i}\right)=\sum_{i=1}^{n} Y_{i} A_{i}$ via the differential specialization $Y_{i} \mapsto f_{i}$.

Part (iii) follows from Proposition 1.31 in 8 .

\section{REFERENCES}

1. A. K. Bhandari and N. Sankaran, Generic differential equations and Picard-Vessiot extensions, Rend. Sem. Mat. Univ. Politec. Torino 52, 4 (1994), 353-358. MR 96f:12007

2. A. Borel, Linear Algebraic Groups, second enlarged edition, Graduate Texts in Mathematics, no. 126, Springer-Verlag, New York, 1991. MR 92d:20001

3. L. Goldman, Specialization and Picard-Vessiot theory, Trans. Amer. Math. Soc. 85 (1957), 327-356. MR 19:384b

4. L. Juan, Principal differential ideals and a generic inverse differential Galois problem for $\mathrm{GL}_{n}$, Comm. Algebra 30, 12 (2002), 6071-6103.

5. M. V. Kondratieva, A. B. Levin, A. V. Mikhalev and E. V. Pankratiev, Differential and Difference Dimension Polynomials, Kluwer Academic Publishers, Dordrecht, 1999. MR 2001c:12006

6. A. Magid, Lectures on differential Galois theory, University Lecture Series, vol. 7, American Mathematical Society, Providence, RI, 1994. MR 95j:12008

7. E. Noether, Gleichungen mit vorgeschriebener Gruppen, Math. Ann. 78 (1918), 221-229.

8. M. Van de Put and M. Singer,Galois theory of linear differential equations, Grundlehren der Mathematischen Wissenschaften [Fundamental Principles of Mathematical Sciences], 328, Springer-Verlag, Berlin, 2003. MR 2004c:12010

9. T. A. Springer, Linear Algebraic Groups, second edition, Progress in Mathematics, vol. 9, Birkhäuser Boston, Boston, MA, 1998. MR 99h:20075

Department of Mathematics and Statistics, Texas Tech University, Box 41042, LubBOCK, TEXAS 79409-1042

E-mail address: ljuan@math.ttu.edu

$U R L:$ http://www.math.ttu.edu/ ${ }^{\prime} l$ juan 